\title{
DESEMPENHO MERCADOLÓGICO NO MERCADO DE ALTA TECNOLOGIA: UMA REVISÃO SISTEMÁTICA
}

Gislayne da Silva Goulart ${ }^{1}$, Alessandra Ferrari Weber, Rafael Barreiros Porto Universidade Federal de Mato Grosso do Sul - UFMS, Mato Grosso do Sul, (Brasil) Universidade de Brasília - UnB, Brasília, (Brasil)

\section{DETALHES DO ARTIGO}

Recebido: 22 de março de 2019

Aceito: 25 de setembro de 2019

Disponível online: 01 de jan. de 2020

Sistema de revisão "Double blind review"

\section{Editor Científico}

Ilan Avrichir

\section{Palavras-chaves:}

Alta tecnologia

Indicadores de desempenho

Mercados dinâmicos

Iramuteq

\begin{abstract}
RESUMO
Objetivo: Analisar a produção científica relativa ao desempenho mercadológico no mercado de alta tecnologia no período de 1997 a 2019.

Método: Foram utilizadas as metodologias de bibliometria e revisão sistemática. O processo de busca foi realizado nas bases Web of Science e SPELL e resultou na seleção de 23 artigos dentro do escopo definido, dentre 82 estudos. Os estudos selecionados foram analisados por meio de estatística descritiva, Classificação Hierárquica Descendente (CHD) e Análise Fatorial de Correspondência (AFC).

Principais resultados: Os resultados apontam um recente interesse sobre a mensuração de desempenho no mercado de alta tecnologia, sobretudo nos mercados emergentes como Taiwan e China, com o uso de indicadores multidimensionais, financeiros e não financeiro. Por meio das análises de CHD e AFC observou-se a formação de quatro Classes - 'Habilidades internas', 'Alianças', 'Foco a jusante' e 'Foco a montante' - cujas temáticas predominantes explicitam os focos de interesse associados ao desempenho mercadológico no mercado de alta tecnologia.

Relevância/originalidade: O estudo inova ao propor um protocolo para realizar análises textuais de artigos científicos com auxílio do software Iramuteq, possibilitando a identificação dos diferentes enfoques dos estudos e agrupamento por semelhanças.

Contribuições: É proposta uma agenda de estudos futuros em função dos resultados e de lacunas sobre desempenho e indicadores utilizados pelas empresas no mercado de alta tecnologia. Sob o prisma gerencial, destaca-se que os indicadores mais utilizados nesse mercado são as vendas, a participação de mercado e o desempenho de novos produtos.
\end{abstract}

\section{Introdução}

Nos últimos anos, o mercado de alta tecnologia vem apresentando um crescimento notório. Esse mercado é caracterizado pelo alto grau de incertezas tecnológicas e do mercado consumidor, volatilidade competitiva, despesas com pesquisa e desenvolvimento (P\&D), rápida obsolescência de produtos, internacionalização das operações e presença de externalidades de rede (Mohr, Sengupta \& Slater, 2010), além de apresentar empresas com maior tendência de orientação para engenharia e foco no produto ao invés de orientação para aspectos mercadológicos (Mohr \& Shooshtari, 2003, Mohr et al., 2010, Vandenbroucke, Knockaert, \& Ucbasaran, 2016). Considerando as inúmeras complexidades que configuram os mercados de alta tecnologia, estudos apontam para um gap de pesquisas de marketing nessa área (Patterson \& Dawes, 1999, Mohr \& Shooshtari, 2003, Mohr et al., 2010, Troung, 2017). Nesse aspecto, destaca-se que há uma crescente importância da mensuração das atividades e dos efeitos do marketing no desempenho empresarial, apontando para que os pesquisadores foquem em estudos voltados às métricas de marketing (Clark \& Ambler, 2001).

Por outro lado, estudos sobre o desempenho organizacional tem apresentado a utilização de variáveis, métodos e indicadores de desempenho diversos, tanto financeiros como não financeiros, associando os resultados desses indicadores à multidimensionalidade e dinamicidade do desempenho organizacional (Gama, 2011). 
Considerando a velocidade que a tecnologia imprimiu aos negócios, novos desafios com relação à medição de desempenho são impostos, principalmente no mercado de alta tecnologia, devido à volatilidade, velocidade e incertezas ligadas a esse tipo de indústria (Chiesa \& Frattini, 2011, Ma, Yang, Yoa, Fisher \& Fang, 2012, Kou \& Lee, 2014).

Dentre as diversas facetas da medição do desempenho pelas empresas estão aquelas associadas ao marketing, ao mercado, às vendas, aos aspectos comerciais e às marcas, sendo na presente pesquisa consideradas todas e englobadas na terminologia 'desempenho mercadológico'.

Neste contexto, o presente estudo tem como questão norteadora: O que vem sendo abordado sobre desempenho mercadológico no mercado de alta tecnologia? Assim, o objetivo principal deste estudo é apresentar um panorama dos estudos relacionados ao desempenho mercadológico no mercado de alta tecnologia, apresentando os resultados de uma revisão sistemática e bibliométrica dos estudos realizados em âmbito internacional e nacional (Brasil) no período de janeiro de 1997 a fevereiro de 2019.

Este trabalho está composto por seções que versam a respeito dos pressupostos teóricos sobre desempenho mercadológico, dos procedimentos metodológicos utilizados e os critérios de seleção dos artigos científicos, e, dos principais resultados que são apresentados e discutidos. Por último, encontram-se as considerações finais e a proposição de uma agenda de pesquisa.

\section{Referencial Teórico}

No que tange à abordagem de desempenho no âmbito mercadológico verificada na literatura, podese citar o desempenho comercial, de marketing, de mercado, de negócios, de vendas e de marcas. Essas terminologias têm sido utilizadas pelos autores para se referir ao desempenho empresarial com enfoque no mercado, tanto em termos financeiros como em ativos intangíveis, como qualidade, percepção, satisfação, dentre outros, sem que haja uma única linha teórica sobre a conceituação consolidada de cada tipo de desempenho mencionado (Varadarajan, 1986, Venkatraman \& Ramanujan, 1986, Szymanski, Troy \& Bharadwaj, 1995, Clark \& Ambler, 2001).

A importância do marketing, bem como, a compreensão e medição de como as atividades de marketing podem influenciar no desempenho da empresa tem sido objeto de diversos estudos nas últimas décadas (por exemplo, Varadarajan, 1986, Aaker, 1996, Clark, 1999, Clark \& Ambler, 2001, Ambler, Kokkinaki \& Puntoni, 2004, O’Sullivan \& Abela, 2007; Gao, 2010, Gama, 2011). A atenção requerida entre as atividades de marketing e o desempenho do negócio é impulsionada pelo desejo de crescimento de vendas, orientação para o mercado e pela responsabilização pelo desempenho (Clark \& Ambler, 2001).

Estudos indicam que "a capacidade de medir o desempenho do marketing tem um impacto significativo no desempenho da empresa, na rentabilidade, no retorno das ações e na estatura do marketing dentro da empresa" (O'Sullivan \& Abela, 2007, p. 79). Eusebio, Andreu e Belbeze (2006) consideram como marketing performance medidas de eficácia de marketing, em outras palavras, o desempenho de marketing seria medições de diversos indicadores que revelem se as ações de marketing foram eficazes. Gao (2010), embasado em outros estudos, define desempenho de marketing como um processo multidimensional, onde a eficácia e a eficiência das atividades de marketing estão relacionadas aos objetivos de mercado, como receitas, crescimento e participação no mercado. Em que pese a existência de diversos estudos sobre desempenho de marketing (Gao, 2010, Guissoni \& Neves, 2013), não há consenso sobre esse conceito (Gao, 2010, Wu, 2011) e várias métricas e indicadores, financeiros e não financeiros, vêm sendo utilizados (Clark, 1999), por não existir uma medida de desempenho consolidada, ou seja, elas variam em função do contexto e relevância (Ambler \& Kokkinaki, 1997, Lau \& Bruton, 2011, Gama, 2011).

Em outro aspecto, a marca na sua amplitude de conceptualização representa para uma empresa um potencial diferencial, fonte de retornos tangíveis e intangíveis, tornando relevante a mensuração do seu desempenho. Segundo Ehrenberg, Uncles e Goodhardt (2004), as vendas de uma marca são determinadas por medidas como quantos clientes compram a marca, com que frequência e também quanto compram de outras marcas. Louro (2000) sintetiza, embasado em outros estudos, que as medidas convencionais de mensuração do desempenho de marca são de notoriedade, associação/diferenciação, qualidade percebida/liderança, lealdade e mercado. Cada medida dessas pode ser composta por diferentes combinações de indicadores dependendo do 
contexto investigado, podendo levar a uma melhor explicação do desempenho da marca.

Outro enfoque utilizado na análise de desempenho no mercado de alta tecnologia foi o desempenho comercial, traduzido pelo saldo da balança comercial entre países, considerando que há uma internacionalização de boa parte da manufatura desse setor, podendo existir a fragmentação das atividades, onde etapas tecnológicas mais complexas são desenvolvidas em uma parte do mundo e outras mais intensivas em mão de obra são executadas por outros países (Rauen \& Furtado, 2014).

Assim, há uma variedade de métricas e indicadores que fazem parte do rol de itens a serem selecionados para medir o desempenho de marketing, mercado, vendas e/ou marca, tais como: market share, vendas, retorno sobre investimento (ROI), retorno sobre capital (ROC), retorno sobre o patrimônio (ROE), retorno sobre ativos (ROA), número de novos produtos, número de clientes, reputação, satisfação do cliente, lucro, valor das ações, aceitação de mercado, brand equity (Varadarajan, 1986, Venkatraman \& Ramanujan, 1986, McKee, Varadarajan \& Pride, 1989, Aaker, 1996, Szymanski et al., 1995, Ambler \& Kokkinaki, 1997, O'Sullivan \& Abela, 2007, Lew, Sinkovics \& Kuivalainen, 2013).

Entretanto, segundo sintetizado por Lau \& Bruton (2011, p. 375) "pode ser problemático medir o desempenho em empresas empreendedoras, em particular as empresas de alta tecnologia". Os autores sugerem a utilização de vários tipos de medidas e citam como exemplo que seria preferível utilizar medidas de vendas do que as de lucro, no caso de empresas jovens de tecnologia, pois as vendas podem ser mais essenciais à sua sobrevivência, e que desempenho no desenvolvimento de novos produtos também é um fator crítico para as empresas de alta tecnologia (Lau \& Bruton, 2011).

Há que se considerar ainda que os indicadores de desempenho podem ser classificados em objetivos e subjetivos. Os indicadores objetivos são aqueles obtidos por meio de dados objetivos e válidos, como dados financeiros e de relatórios empresariais (dados secundários). Devido ao difícil acesso a esses dados (Bruton \& Rubanik, 2002), pesquisadores vem testando e validando também como indicadores objetivos os dados resolutos informados pelos respondentes (self-report). Já os indicadores subjetivos (de percepção) são aqueles cujas informações geralmente são coletadas por meio de escalas (Dess \& Robinson, 1984, Venkatraman \& Ramanujam, 1987, Baker, Gibbons \& Murphy, 1994, Perin \& Sampaio, 1999). Na área mercadológica, pesquisadores também procuram testar, comparar e validar indicadores objetivos e subjetivos, bem como criar modelos de mensuração de desempenho da empresa utilizando indicadores subjetivos e/ou ambos. Todavia, ressaltam que podem existir gaps entre medidas subjetivas, e o que de fato ocorre, e demonstram que não há um padrão entre os possíveis indicadores em diferentes mercados (Pelham \& Wilson, 1996, Dawes, 1999, Harris, 2001, Wall et al., 2004, Morgan, Vorhies \& Mason, 2009, Santos \& Brito, 2012, Chen et al., 2017, Bayraktar, Hancerliogullari, Cetinguca \& Calisir, 2016). Dito isto, surgem novos questionamentos: Quais composições dos indicadores de desempenho estão sendo utilizadas na mensuração do desempenho mercadológico no mercado de alta tecnologia? Qual tipo de medida e fonte dos dados são mais consideradas?

Com base no exposto, será considerado no presente estudo que o desempenho comercial, de marketing, de mercado, de vendas e de marca podem ser sintetizados como 'desempenho mercadológico' possibilitando, assim, uma maior amplitude quando se pretende investigar o cenário recente das pesquisas sobre a temática no contexto do mercado de alta tecnologia.

\section{Procedimentos E Métodos}

O presente estudo é de cunho quali-quantitativo, sendo utilizado as metodologias de revisão sistemática, que constitui-se em um método estruturado para a identificação de estudos relevantes sobre uma temática em particular (Rother, 2007; Dybå \& Dingsøyr, 2008); e a bibliometria que auxilia na análise da produção científica, possibilitando delinear um panorama do desenvolvimento e comportamento de uma área do conhecimento (Araújo \& Alvarenga, 2011; Vanti, 2002). Foi realizado um levantamento da produção acadêmica do período de janeiro de 1997 a fevereiro de 2019, optando-se por efetuar a busca em duas bases de dados: no ISI Web of Science - cujo processo de busca acessa artigos publicados em mais de 12.000 periódicos indexados e classificados com fator de impacto no Journal Citation Reports (JCR), bem como artigos que estão disponíveis em outras bases, como o ProQuest, Scopus e Wiley (Pereira, Carvalho 
\& Rotondaro, 2013); e no Spell - seu processo de busca permite acesso aos principais periódicos nacionais (brasileiros) das áreas de Administração, Contabilidade e Turismo.

As coletas dos dados foram realizadas na base ISI Web of Science, utilizando-se os termos-chaves: "brand performance AND high-tech", "brandperformance AND high-tech*", "market*performance AND high-tech*", "sales-performance AND high-tech*", "commercial-performance AND high-tech*" e na base SPELL, onde foram utilizados os termos-chaves: "desempenho de marca e alta tecnologia", "desempenho de mercado e alta tecnologia", "desempenho de vendas e alta tecnologia", "desempenho de marketing e alta tecnologia", "desempenho comercial e alta tecnologia". Em um primeiro momento, foram considerados os estudos teórico-empíricos que possuíam algum desses termos-chaves em alguma parte do texto. No total, foram encontrados 82, desses 72 foram publicados em periódicos internacionais e 10 em periódicos nacionais. Em um segundo momento, foi realizada a leitura dos títulos e resumos dos estudos com a intenção de averiguar o alinhamento deles com o propósito deste estudo, resultando em 38 artigos. Posteriormente à leitura na íntegra dos artigos, obteve-se 23 artigos (desse total apenas um é nacional), os quais compõem a amostra de análise do presente estudo, considerando o cumprimento dos seguintes critérios: os dados da pesquisa eram provenientes do mercado de alta tecnologia e os indicadores de mensuração foram explicitados. A Figura 1 esquematiza os procedimentos relatados de seleção de estudos.

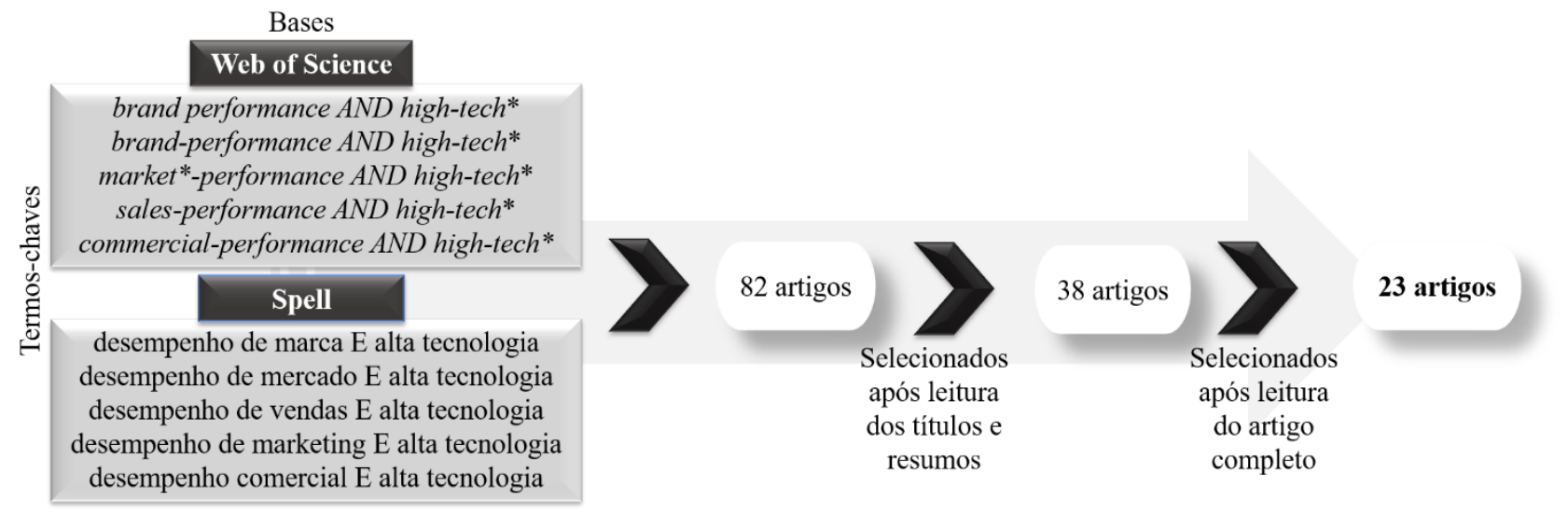

Figura 1: Protocolo da seleção de estudo

Para se proceder à revisão bibliométrica dos artigos selecionados, foi utilizado o 'Package Bibliometrix' do software $\mathrm{R}$ que possibilitou a caracterização da amostra. Em seguida para o propósito do estudo propôs-se um protocolo para realizar análises textuais com auxílio do software IRAMUTEQ 0.7 (Interface de $R$ pour les Analyses Multidimensionnelles de Textes et de Questionnaires) (Ratinaud, 2014). Para isso foi necessário a construção de um corpus (conjunto de textos que se pretende analisar) composto pelo título, resumo e palavras-chaves dos estudos selecionados. Para uma melhor congruência dos resultados foram também acrescidas ao corpus cargas de representatividade dos estudos, ou seja, após leitura sistemática dos artigos foram identificados os temas gerais e microtemas e, esses foram acrescidos ao corpus para que os contextos semânticos formados se tornassem mais representativos dos estudos. Bem como, foram consideradas como variáveis descritivas: área geográfica de realização do estudo (países/região); natureza do estudo (qualitativo, quantitativo e qualiquanti); recorte temporal (transversal, longitudinal e ambos); e tipo de indicador de desempenho adotado em cada estudo (indicadores objetivos, indicadores subjetivos e ambos). O protocolo da composição do corpus para análise de artigos científicos pode ser verificado na Figura 2. 


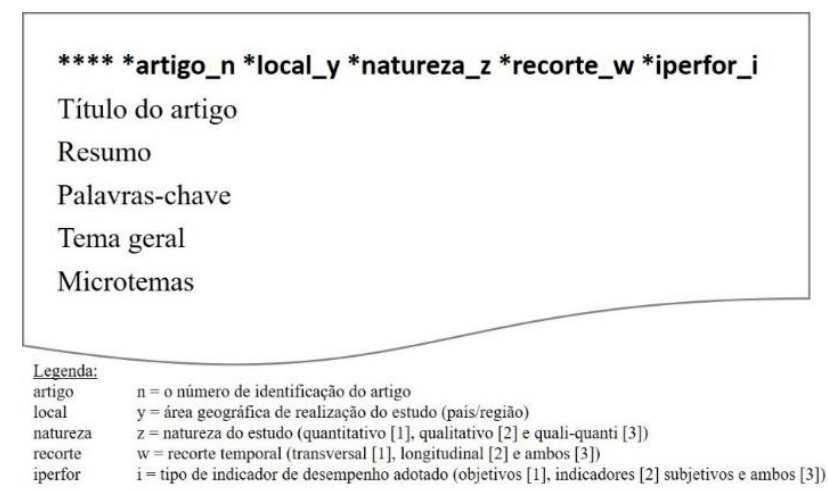

Figura 2. Protocolo da composição do corpus para análise de artigos científicos Fonte: Elaborado pelos autores

Após a verificação da validação do corpus, foram realizadas as análises de nuvem de palavras, a Classificação Hierárquica Descendente (CHD) e a Análise Fatorial de Correspondência (AFC). A nuvem de palavras permite uma visualização lexicográfica das palavras mais representativas do corpus investigado. Dado que o desempenho mercadológico foi investigado em um mercado específico, considerou-se relevante realizar uma CHD. A CHD oportuniza uma análise lexical e agrupamento de vocabulário semelhante entre si e distinto dos segmentos de texto dos outros grupos, por meio de diversos testes do tipo $X^{2}$ (ver Reinert, 1983,1990). A análise dos segmentos de texto é apresentada por meio de um dendograma da CHD, que possibilita a verificação das relações entre as classes, em outras palavras, essa análise possibilita conhecer estatisticamente o panorama de como os estudos que compõem a amostra se assemelham ou se distanciam entre si. Com base nas classes formadas a partir da CHD, o programa Iramuteq realiza a AFC, essa análise permite a representação de clusters em um plano cartesiano formado pelos segmentos de textos mais característicos de cada classe e variáveis associadas a cada uma delas, evidenciando as interrelações existentes (Camargo, 2005, Camargo \& Justo, 2013). Todas essas análises em conjunto possibilitaram um melhor entendimento do que está sendo abordado sobre desempenho mercadológico no âmbito do mercado de alta tecnologia.

\section{Análise e Discussão dos Resultados}

Para um entendimento mais congruente dos resultados são apresentados na Tabela 1, os 23 estudos que compõem a bibliometria, tipo de coleta de dados e as respectivas amostras de cada estudo. Cabe salientar que apenas um estudo brasileiro integrou a amostra, revelando a pouca exploração sobre o assunto pela pesquisa nacional.

\section{Tabela 1 Produção científica analisada}

\begin{tabular}{|c|c|c|c|c|c|}
\hline Autores & & Amostra & Autores & & mostra \\
\hline Li \& Atuahene-Gima (1999) & $\mathrm{T}$ & $114_{128 p}$ & Patel (2014) & $\mathrm{L}$ & $305^{\mathrm{E}}$ \\
\hline Aaker \& Jacobson (2001) & $\mathrm{L}$ & $9^{E_{206 f}}$ & Rauen \& Furtado (2014) & $\mathrm{L}$ & $16^{P}$ \\
\hline Atuahene-Gima \& Li (2002) & $\mathrm{T}$ & $150^{E_{347 v}}$ & Kou \& Lee (2015) & $\mathrm{T}$ & $242^{\mathrm{E}}$ \\
\hline Chang, Lin, \& Sheu (2002) & $\mathrm{T}$ & $87^{\mathrm{E}}$ & Kou, Lee \& Wei (2015) & $\mathrm{T}$ & $29 \mathrm{E}_{2376}$ \\
\hline O'Sullivan \& Abela (2007) & $\mathrm{L}$ & $312^{G} 176 f$ & Oh, Cho \& Kim (2015) & $\mathrm{L}$ & $2496^{\mathrm{E}}$ \\
\hline O'Sullivan, Abela \& Hutchinson (2009) & $\mathrm{L}$ & $157^{G} 128 f$ & Wang, Chen, Yu \& Hsiao (2015) & $\mathrm{L}$ & $1086^{\mathrm{AF}}$ \\
\hline Chiesa \& Frattini (2011) & $T$ & $8^{\mathrm{IL}}$ & Vandenbroucke et al. (2016) & L & $80^{\mathrm{E}}$ \\
\hline Wu (2011) & $\mathrm{T}$ & $172^{\mathrm{E}}$ & Moghaddam, Bosse \& Provance (2016) & $L$ & $151^{\mathrm{E}}$ \\
\hline Lau \& Bruton (2011) & $\mathrm{T}$ & $150^{\mathrm{E}}$ & Nguyen, Yu, Melewar \&Gupta (2016) & $\mathrm{T}$ & $182^{\mathrm{E}}$ \\
\hline Ma et al. (2012) & $\mathrm{T}$ & $142^{\mathrm{E}}$ & Wu \& Lin (2016) & $\mathrm{T}$ & $312^{R}$ \\
\hline Lew et al. (2013) & $\mathrm{T}$ & $110^{\mathrm{E}}$ & Mukarram et al. (2018) & L & $121^{\mathrm{E}}$ \\
\hline Fuertes-Callén \& Cuéllar-Fernández (2014) & $\mathrm{L}$ & $142^{\mathrm{E}}$ & & & \\
\hline
\end{tabular}


Apesar do período pesquisado englobar 22 anos, de 1997 a 2019, a primeira publicação dentro do foco de desempenho mercadológico no mercado de alta tecnologia, de acordo com os critérios de busca, somente ocorreu em 1999, sendo que na primeira década subsequente, compreendida entre os anos de 2000 a 2009, ocorreram 5 publicações de estudos, correspondendo a $21,7 \%$ do total dos artigos analisados. De 2010 a 2018, publicaram-se 17 artigos (73,9\%), sendo os anos de 2015 e 2016 os mais representativos, com 4 artigos cada. Esses dados sugerem uma atenção recente a respeito da pesquisa sobre o desempenho mercadológico no mercado de alta-tecnologia.

Do total da amostra, 21 estudos tiveram natureza quantitativa, representando $91,3 \%$ da amostra, um estudo teve a natureza qualitativa $(4,3 \%)$ e outro teve natureza quali-quantitativa $(4,3 \%)$. Os estudos

QUANTIDADE DE PUBLICAÇÕES POR ANO
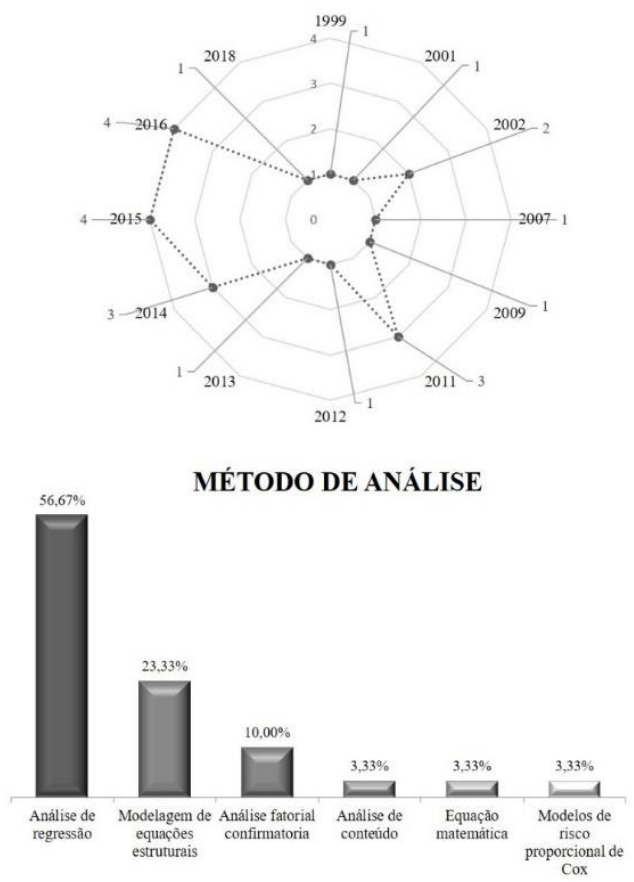

RECORTE TEMPORAL

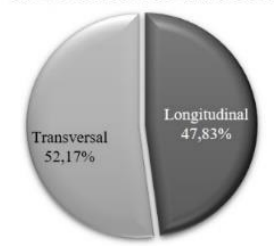

Figura 3. Caracterização dos estudos tiveram predominância do recorte temporal transversal (52,2\%), ou seja, os dados coletados para análises em sua maioria foram por meio de survey. Dentre dos métodos de análise de dados mais adotados pelos pesquisadores, destaca-se o uso da análise de regressão, em seus diversos modelos, com incidência em $56,7 \%$ dos estudos. Outros métodos de análise identificados foram a modelagem de equações estruturais (23,3\%), a análise fatorial confirmatória (10\%), a análise de conteúdo, a equação matemática e o modelo de risco proporcional de Cox, sendo os três últimos métodos com 3,3\% de participação cada um. As análises estatísticas estiveram presentes em 95,24\% dos casos estudados. As representações gráficas relativas à quantidade de publicações de artigos por ano, à natureza do estudo, ao tipo de recorte temporal e aos métodos de análises utilizados, encontram-se na Figura 3.

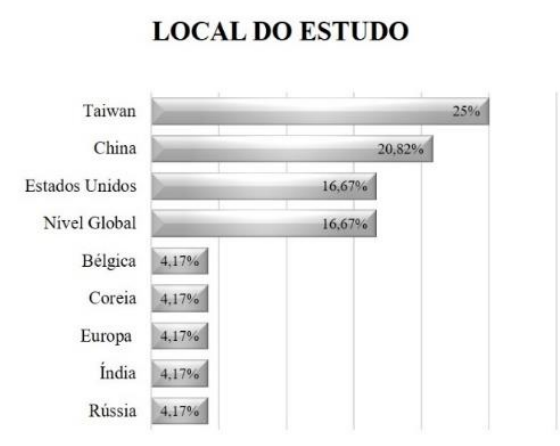

\section{NATUREZA DO ESTUDO}

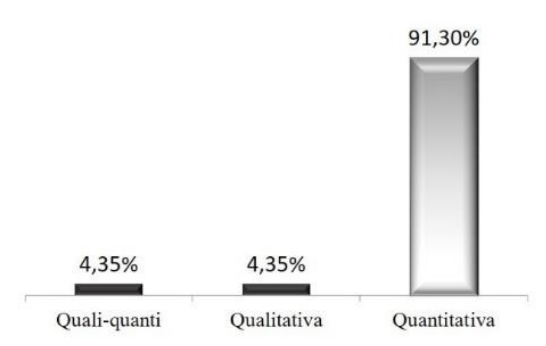

\section{INDICADOR DE DESEMPENHO}

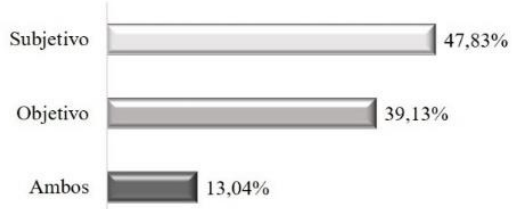


As áreas geográficas, cujas empresas de alta tecnologia foram objeto de análise quanto ao desempenho mercadológico, estão representadas na Figura 3, na qual se pode observar uma concentração dos estudos em países da Ásia, tais como Taiwan, China e Coreia, com $48 \%$ dos estudos, seguida do nível global (empresas de países de continentes diferentes) e dos Estados Unidos, cujas representatividades foram de 16\%. Ressalta-se que Taiwan têm se destacado no cenário mundial como um dos principais fabricantes e parceiros estratégicos de produção no mercado de alta tecnologia (Kou \& Lee, 2015).

A mensuração do desempenho mercadológico no mercado de alta tecnologia tem apresentado variação quanto à composição dos indicadores de desempenho utilizados, bem como o uso de mais de uma dimensão pelos autores dos estudos para classificá-los, conforme pode ser verificado na Tabela 2. A variedade e multidimensionalidade dos indicadores de desempenho identificados se alinham aos relatos teóricos de Venkatraman e Ramanujan (1986), Bruton e Rubanik (2002), Gao (2010) e Gama (2011), sendo preferível utilizar, nesse mercado, várias medidas de desempenho (Lau \& Bruton, 2011).

Entretanto, foi possível identificar que as vendas, a participação de mercado (market share) e a preocupação com a aferição de desempenho de novos produtos, com a incidência de medição em 52,17\%, $43,48 \%$ e $26,09 \%$ dos estudos, respectivamente, são indicadores relevantes no mercado de alta tecnologia, corroborando os aspectos teóricos ressaltados por Lau e Bruton (2011), sobre esses fatores serem críticos para a sobrevivência de empreendimento de alta tecnologias. Há uma predominância na utilização de indicadores subjetivos para se medir o desempenho mercadológico, com representatividade em 47,83\% dos casos investigados, seguidos de indicadores objetivos $(39,13 \%)$ e de ambos, com 13,04\%. A incidência de medição de indicadores por meio de escala de " $n$ " pontos ocorreu em 12 estudos (52,17\%), tendo a mesma quantidade com a medição por meio de dados de fontes secundárias, ou seja, em $86,96 \%$ dos estudos houve o uso de uma ou ambas os tipos de fonte de dados citadas.

Tabela 2

Indicadores de desempenho na perspectiva dos autores dos estudos

\begin{tabular}{|c|c|c|c|c|}
\hline $\begin{array}{l}\text { Classificação do } \\
\text { Desempenho }\end{array}$ & Autores & Composição dos indicadores de desempenho & $\begin{array}{l}\text { Tipo de } \\
\text { Medida }\end{array}$ & $\begin{array}{c}\text { Fonte do } \\
\text { Dados }\end{array}$ \\
\hline $\begin{array}{l}\text { Benefício } \\
\text { percebido pelo } \\
\text { consumidor e } \\
\text { aceitação de } \\
\text { mercado } \\
\end{array}$ & Patel (2014) & $\begin{array}{c}\text { Número de serviços bancários percebidos como sendo } \\
\text { benéficos pelos clientes. } \\
\text { Número de usuários registrados on-line do site transacional. }\end{array}$ & Objetiva & DS \\
\hline \multirow{2}{*}{$\begin{array}{l}\text { Comercialização } \\
\text { da inovação }\end{array}$} & \multirow[b]{2}{*}{ Chiesa \& Frattini (2011) } & Volume de Vendas. & \multirow[b]{2}{*}{ Ambas } & \multirow[b]{2}{*}{$\mathrm{DS}$ e $\mathrm{AH}$} \\
\hline & & $\begin{array}{l}\text { Atitude dos primeiros adotantes (positiva ou negativa) e } \\
\text { sucesso na rede de adoção. }\end{array}$ & & \\
\hline Desempenho & Wu (2011) & $\begin{array}{c}\text { Número de patentes, taxa de novos produtos de sucesso e } \\
\text { taxa de introdução de novos produtos, todos relativos ao } \\
\text { principal competidor, e primeiro a entrar no mercado com a } \\
\text { nova aplicação. }\end{array}$ & Subjetiva & $A E$ \\
\hline $\begin{array}{c}\text { Desempenho } \\
\text { comercial }\end{array}$ & Rauen \& Furtado (2014) & Saldo comercial = exportações sobre as importações. & Objetiva & DS \\
\hline \multirow{6}{*}{$\begin{array}{l}\text { Desempenho da } \\
\text { empresa }\end{array}$} & $\begin{array}{l}\text { O'Sullivan \& Abela } \\
\text { (2007) }\end{array}$ & \multirow{2}{*}{$\begin{array}{l}\text { Crescimento de vendas, market share, rentabilidade, } \\
\text { retorno sobre ativos (ROA) e retorno sobre ações. }\end{array}$} & \multirow[t]{2}{*}{ Ambas } & \multirow[t]{2}{*}{ AE e DS } \\
\hline & O'Sullivan et al. (2009) & & & \\
\hline & Lau \& Bruton (2011) & $\begin{array}{c}\text { Desempenho de vendas (crescimento de vendas e market } \\
\text { share), desempenho de novos produtos (novos produtos no } \\
\text { mercado e despesa de P\&D), desempenho eficiente da } \\
\text { produção (capacidade de produção e eficiência de } \\
\text { produção). }\end{array}$ & Subjetiva & $\mathrm{AE}$ \\
\hline & Oh et al. (2015) & Vendas e lucro operacional. & Objetiva & DS \\
\hline & Wang et al. (2015) & $\begin{array}{c}\text { Retorno sobre o patrimônio (ROA), retorno sobre o } \\
\text { patrimônio líquido (ROE) e Q da Tobin (Tobinq). }\end{array}$ & Objetiva & DS \\
\hline & $\begin{array}{l}\text { Mukarram, Saeed, } \\
\text { Hammoudeh \& Raziq } \\
\text { (2018) }\end{array}$ & $\begin{array}{l}\text { Medido pelo Tobin'sQ (relação entre o valor de mercado dos } \\
\text { ativos totais da empresa e seu valor de reposição) }\end{array}$ & Objetiva & DS \\
\hline \multirow{2}{*}{$\begin{array}{l}\text { Desempenho de } \\
\text { marketing }\end{array}$} & Kou \& Lee (2015) & \multirow{2}{*}{$\begin{array}{l}\text { Crescimento médio de market share, crescimento médio do } \\
\text { volume de vendas e crescimento médio de venda (U\$). }\end{array}$} & \multirow{2}{*}{ Subjetiva } & \multirow{2}{*}{$\mathrm{AE}$} \\
\hline & Kou et al. (2015) & & & \\
\hline
\end{tabular}




\begin{tabular}{|c|c|c|c|c|}
\hline \multirow{5}{*}{$\begin{array}{l}\text { Desempenho de } \\
\text { mercado }\end{array}$} & Lew et al. (2013) & $\begin{array}{c}\text { Crescimento de vendas, market share, número de novos } \\
\text { produtos, número de novos clientes, aumento da reputação } \\
\text { e desempenho geral. }\end{array}$ & Subjetiva & AE \\
\hline & $\begin{array}{l}\text { Fuertes-Callén \& Cuéllar- } \\
\text { Fernández (2014) } \\
\end{array}$ & $\begin{array}{l}\text { Número de novos clientes, número de renovação de } \\
\text { contrato e market share. }\end{array}$ & Objetiva & DS \\
\hline & $\begin{array}{c}\text { Moghaddam et al. } \\
(2016)\end{array}$ & Valoração da empresa (Firm valuation). & Objetiva & DS \\
\hline & Nguyen et al. (2016) & $\begin{array}{l}\text { Crescimento de mercado, market share, lucro, ROI e } \\
\text { satisfação do cliente. }\end{array}$ & Subjetiva & AE \\
\hline & $\begin{array}{l}\text { Vandenbroucke et al. } \\
\text { (2016) }\end{array}$ & $\begin{array}{l}\text { Tempo para o lançamento do primeiro produto e número de } \\
\text { produtos. }\end{array}$ & Objetiva & DQ e DS \\
\hline \multirow{4}{*}{$\begin{array}{l}\text { Desempenho de } \\
\text { novo produto }\end{array}$} & $\begin{array}{l}\text { Li \& Atuahene-Gima } \\
\text { (1999) }\end{array}$ & $\begin{array}{l}\text { Na dimensão desempenho de mercado (satisfação interna, } \\
\text { qualidade do produto, volume de vendas, aceitação do } \\
\text { produto, market share e lucro). } \\
\text { Na dimensão pontualidade no desenvolvimento do produto. }\end{array}$ & Subjetiva & $\mathrm{AE}$ \\
\hline & Ma et al. (2012) & $\begin{array}{l}\text { Inovação, velocidade de entrada do produto no mercado e } \\
\text { desempenho de mercado (satisfação interna com volume de } \\
\text { vendas, desempenho do produto no mercado e retorno } \\
\text { financeiro). }\end{array}$ & Subjetiva & $\mathrm{AE}$ \\
\hline & Kou \& Lee (2015) & \multirow{2}{*}{ Volume de vendas, lucro e satisfação dos clientes. } & \multirow{2}{*}{ Subjetiva } & \multirow{2}{*}{ AE } \\
\hline & Kou et al. (2015) & & & \\
\hline $\begin{array}{l}\text { Desempenho de } \\
\text { vendas }\end{array}$ & $\begin{array}{l}\text { Atuahene-Gima \& Li } \\
\text { (2002) }\end{array}$ & $\begin{array}{l}\text { Market share, volume de vendas, vendas de novo produto e } \\
\text { alcance da meta de vendas. }\end{array}$ & Subjetiva & $\mathrm{AE}$ \\
\hline $\begin{array}{l}\text { Desempenho do } \\
\text { negócio }\end{array}$ & Chang et al. (2002) & Taxa de lucro líquido e taxa de crescimento de vendas. & Subjetiva & $\mathrm{DQ}$ \\
\hline $\begin{array}{l}\text { Desempenho } \\
\text { financeiro }\end{array}$ & Aaker \& Jacobson (2001) & Retorno contábil e retorno de ações. & Objetiva & DS \\
\hline $\begin{array}{l}\text { Desempenho } \\
\text { tecnológico }\end{array}$ & $\begin{array}{l}\text { Vandenbroucke et al. } \\
\qquad(2016)\end{array}$ & Tempo para a primeira patente e número de patentes. & Objetiva & DQ e DS \\
\hline $\begin{array}{l}\text { Intensidade } \\
\text { tecnológica }\end{array}$ & Rauen \& Furtado (2014) & P\&D (gasto em P\&D) / VBP (Valor Bruto de Produção) & Objetiva & DS \\
\hline $\begin{array}{l}\text { Valor percebido e } \\
\text { lealdade à marca }\end{array}$ & Wu \& Lin (2016) & $\begin{array}{l}\text { Lealdade à marca = intenção de compra, fidelidade e } \\
\text { compromisso com a marca; } \\
\text { Valor percebido pelo cliente = custo/benefício da marca. }\end{array}$ & Subjetiva & $\mathrm{AE}$ \\
\hline
\end{tabular}

AE - Avaliação por meio de escala; AH - Avaliação histórica; DQ - Dados obtidos por questionário; DS - Dados Secundários

A verificação da representatividade do que os estudos abordam em comum foi possível por meio do método de nuvem de palavras. A formação lexicográfica das palavras com maior representatividade (Figura 4) são: 'empresa, inovação, alta tecnologia, mercado, produto, efeito e desempenho'. Esse resultado revela que a amostra de estudos é emblemática ao contexto proposto de investigação.

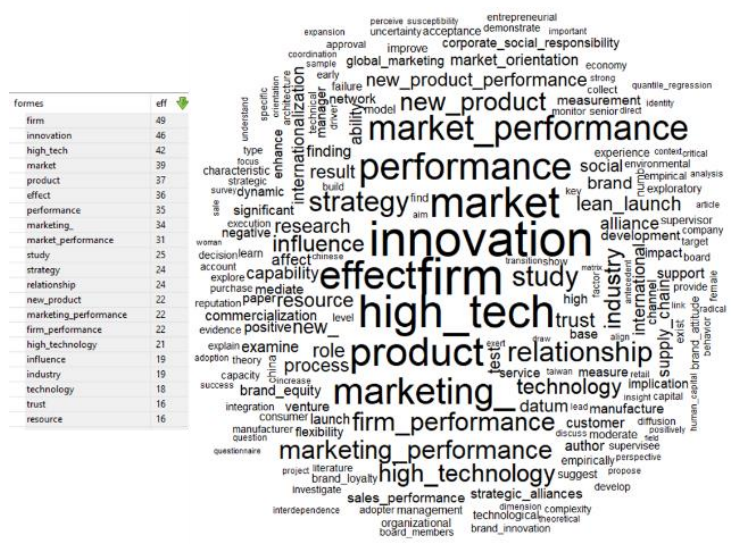

Figura 4: Nuvem de palavras

Com a intenção de apresentar um panorama dos estudos relacionados ao desempenho mercadológico no mercado de alta tecnologia, foi realizada CHD, considerando cada estudo como uma unidade de contexto inicial (UCI). Desse modo, o corpus foi composto por 23 $\mathrm{UCls}$, que deram origem a 107 unidades de contexto elementar (UCEs). A taxa de UCEs retida para análise (o quanto é explicado dos resultados) foi significativa (78,5\%), e foram consideradas como variáveis significativas para análise as variáveis ativas (palavras lematizadas) e descritivas com $X^{2} \geq 3,84$ ( $p$-valor $\leq 0,05$ ). Por meio do tratamento estatístico CHD, obteve-se 4 classes de 
segmentos de textos mais semelhantes correlacionados com as variáveis descritivas. Foi possível identificar como os estudos melhor se aproximam ou se distanciam, mesmo abordando o desempenho mercadológico no contexto investigado (Figura 5). A partir da CHD foi realizada a AFC que proporcionou, de forma mais dinâmica, a compreensão das relações mais marcantes de cada agrupamento de variáveis em um plano fatorial, (Figura 6).

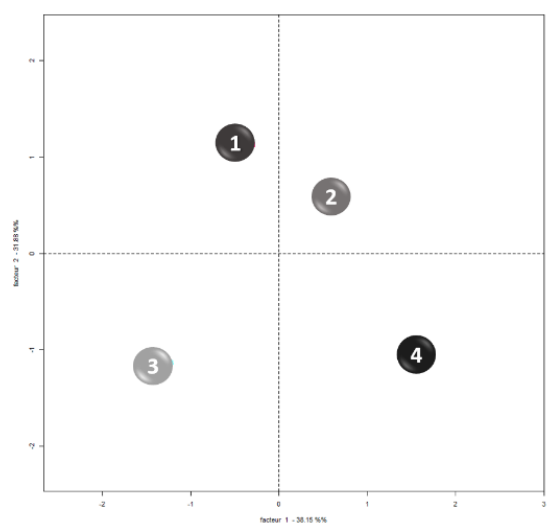

Figura 5: Localização das classes no plano fatorial

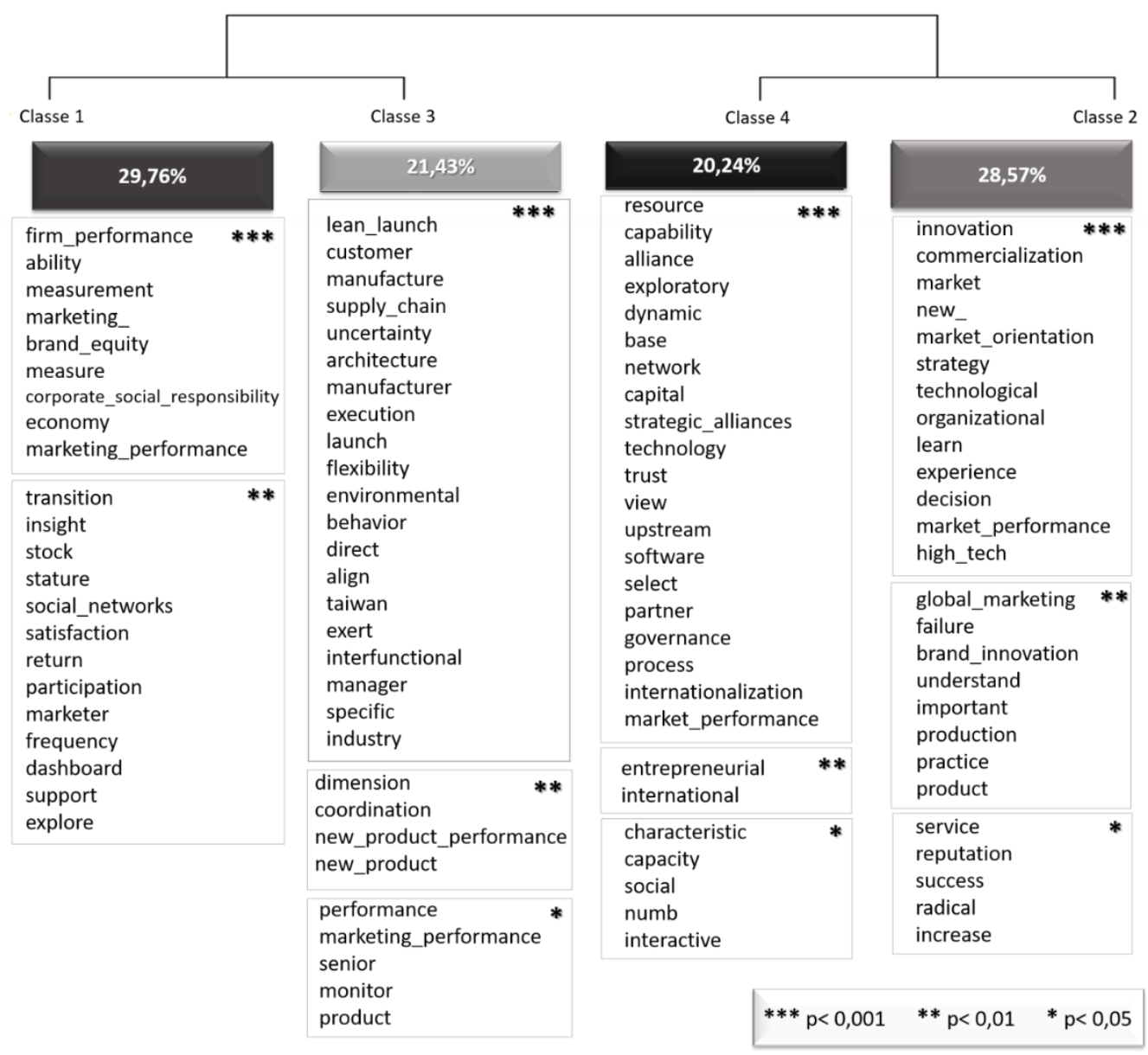

Figura 6: Dendograma da amostra investigada

Cabe salientar que, devido à amostragem, alguns estudos podem não ser citados nas análises, por não apresentarem grau de significância nos testes estatísticos, dado que em primeiro plano foi considerado o agrupamento de contextos semânticos. A análise das modalidades que melhor contribuíram para a formação dos eixos fatoriais permitiu que oposições distintas em relação às outras temáticas que estão sendo abordadas nos estudos fossem identificadas. Cabe acrescentar que tais 
oposições devem ser compreendidas como diferenças no enfoque para o contexto estudado. Assim, uma maior atenção deve ser dada às oposições das classes: 1 e 4; e 3 e 2.

Em relação a "Classe 1 - Habilidades internas", percebe-se que é a maior em representatividade $(29,76 \%)$ e está em quadrante oposto à Classes 4 . É melhor representada pelos estudos de Li e AtuaheneGima (1999) ${ }^{* * *}$, Aaker e Jacobson (2001)*, O'Sullivan e Abela (2007) ${ }^{* * *}$, O'Sullivan et al. (2009) ${ }^{* * *}$, Lau e Bruton (2011) ${ }^{* * *}$, Wang et al. (2015) ${ }^{* * *}$, estudo qualiquantitativo, estudos realizados na China e Rússia*** $\left.^{* *} 100 \%\right)$ e Europa*(62,5\%). O foco centra-se em investigar relações causais entre desempenho e outras variáveis. Os desempenhos investigados segundo os autores foram de novos produtos, de mercado (Li \& Atuahene-Gima, 1999), financeiro (Aaker \& Jacbson, 2001) e da empresa (O'Sullivan \& Abela, 2007, O'Sullivan et al., 2009, Lau \& Bruton, 2011, Wang et al., 2015). Os estudos tendem a investigar o desempenho obtido relacionado à outras medições internas, por exemplo, pontualidade de desenvolvimento de produtos, capacidade de produção, formalização de projeto, poder departamental, satisfação do CEO, geração de relatórios de marketing, orientação estratégica, desenvolvimento corporativo e responsabilidade social.

A “Classe 4 - Alianças" é a menor classe (20,24\%) e melhor representada pelos estudos $\mathrm{Ma}$ et al. $(2012)^{* * *}$, Lew et al. (2013) ${ }^{* * *}$, Moghaddam et al. $(2016)^{* * *}$, estudos com recorte temporal longitudinal $^{* *}(36,11 \%)$ e de área geográfica de estudo de nível global ${ }^{* *}(47,06 \%)$. Esse grupo tem um maior foco em investigar o desempenho de mercado relacionado com alianças estratégicas, capacidade dinâmica, gerenciamento de recursos (estratégicos, tecnológicos e de marketing), relação com parceiros e internacionalização. Em outras palavras, maior foco no mercado e na expansão dos negócios.

Na análise da oposição fatorial das classes 1 e 4 em função do contexto léxico que representam, pode-se observar que a Classe 1 indica para estudos cujo foco no desempenho centra-se em mensurações que apontem relação com um maior enfoque interno. Enquanto que a "Classe 4" um maior foco é dado ao mercado, mais especificamente na expansão dos negócios/internacionalização.

A "Classe 2 - Foco a jusante" (28,57\%) é melhor representada pelos estudos de Chiesa e Frattini
$(2011)^{* * *}$, Wu $(2011)^{*}$, Fuertes-Callén e CuéllarFernández (2014) ${ }^{* * *}$, Oh et al. (2015) ${ }^{* * *}$, Nguyen et al. $(2016)^{* * *}$, Vandenbroucke et al. (2016) ${ }^{*}$, por estudo de natureza qualitativa*** $(100 \%)$, estudos realizados na Coreia*** $(100 \%)$ e na Bélgica* $(100 \%)$. A classe tem foco significativo no mercado, mais especificamente em 'orientação de mercado' e 'desempenho de mercado', e em 'inovação'. Estando mais voltada para os recursos de marketing, estratégias de marketing, comercialização e sucesso/reputação de inovações/novos produtos. Os estudos abordam o mercado de alta tecnologia, desde uma forma mais ampla até as mais específicas, tais como start-up e indústrias de serviço, e apresentam menção direta e/ou indiretamente ao consumidor final (consumer) para tratar de desempenho. Alguns estudos relacionam de modo geral, diretamente (Nguyen et al., 2016) e indiretamente (Chiesa \& Frattini, 2011), a marca com a mensuração de desempenho.

Em relação à "Classe 3 - Foco a montante" que está em um quadrante oposto à Classe 2, é constituída por $21,43 \%$ da amostra, é mais bem representada pelos estudos de Chang et al. (2002) ${ }^{* * *}$, Kou e Lee $(2015)^{* * *}$, Kou et al. (2015) $)^{* * *}$, estudos realizados em Taiwan*** (70,83\%), com recorte temporal transversal ${ }^{* * *}(37,5 \%)$ e todos os estudos dessa classe são caracterizados por adotar indicadores $^{* *}$ de desempenho subjetivos. Esse cluster agrupa estudos voltados para a indústria de manufatura e supply chain, a preocupação com as incertezas ambientais do mercado de alta tecnologia, bem como possui foco nas parcerias e clientes (customer). Há estudo (Chang et al., 2002) que relaciona a flexibilidade de fabricação com o desempenho de negócios e vendas, enquanto outros estudos (Kou \& Lee, 2015, Kou et al., 2015) relacionam lean launch (lançamento ágil/de baixo custo) com agregação de valor para o desempenho de novos produtos e marketing.

Na análise da oposição fatorial da Classe 2 e Classe 3, em função do contexto léxico que representam, pode-se observar que na primeira classe o foco de desempenho é mais voltado para o mercado e consumidor final, enquanto que a segunda classe aponta para estudos cujo foco no desempenho centra-se em mensurações que aponte relação com a produção.

Apesar de não ter sido possível identificar um padrão nos tipos de classificação dos indicadores utilizados no mercado de alta tecnologia, há uma 
maior incidência de uso de alguns termos, como o "desempenho da empresa" dentro da classe "Habilidades internas", o termo "desempenho de mercado" dentro das classes "Aliança" e "Foco a jusante" e o termo "desempenho de novo produto" na classe "Foco a montante", o que pode indicar uma tendência de consenso no tipo de classificação dos desempenhos por focos de interesse.

\section{Considerações Finais}

Este estudo procurou contribuir para a produção acadêmica no campo sobre o desempenho mercadológico, com foco especial no mercado de alta tecnologia, abrangendo estudos no período de 1997 a 2019. Cabe ressaltar que este estudo não pretendeu esgotar a literatura alusiva ao assunto no recorte temporal investigado, em razão de ter se restringido aos periódicos indexados na base Web of Science, por serem classificados com fator de impacto no Journal Citation Reports (JCR) e na base nacional Spell.

A investigação possibilitou identificar, com base nos termos-chaves utilizados, que apenas um estudo, publicado em periódico indexado na base nacional Spell, aborda a proposta, revelando um gap de estudos sobre a temática no Brasil. Os resultados permitiram a constatação do crescente interesse no desenvolvimento de estudos voltados à mensuração do desempenho em empresas de alta tecnologia, com destaque para países emergentes como Taiwan e China, que despontam no mercado internacional de tecnologia; e para o uso multidimensional de indicadores para a mensuração do desempenho mercadológico das empresas, bem como focos temáticos diversos de análise desse mercado associados ao marketing. Não foi identificado um consenso entre os autores na classificação, tipos e dimensões relativas à mensuração do desempenho mercadológico, existindo uma miríade de indicadores financeiros (tais como volume de vendas, lucros, valor de ações, ROE e ROA, etc) e indicadores não financeiros (participação de mercado, satisfação com a qualidade do produto, satisfação de clientes, eficiência do processo de produção e inovação, sucesso em parcerias, etc), em que pese a maior frequência na medição dos indicadores ligados às vendas, market share e desempenho de novos produtos, sinalizando a importância desses fatores no mercado de alta tecnologia.

O protocolo proposto de análise textual se demonstrou válido, considerando que as análises de
CHD e AFC resultaram na formação de quatro Classes - 'Habilidades internas', 'Alianças', 'Foco a jusante' e 'Foco a montante' - que, respectivamente, abordam: a relação da medição das atividades internas de marketing com o desempenho de mercado e da empresa; o gerenciamento de parcerias e recursos para a expansão dos negócios e internacionalização; a orientação para o mercado e inovação de produtos com foco no consumidor final; e por fim, a agregação de valor à cadeia de suprimento pela eficiência da produção. Os resultados coligidos reiteram o potencial para novos estudos sobre desempenho mercadológico, especificamente no contexto do mercado de alta tecnologia, os quais se traduzem em subsídios para que os gestores implementem medições mais robustas de desempenho.

Observa-se, com base nos estudos analisados, consistente potencial de continuidade de estudos nessa área devido ao reduzido número trabalhos encontrados sobre desempenho mercadológico, principalmente no âmbito nacional (Brasil). Mais especificamente, observou-se lacuna em relação à investigação do desempenho da marca nesse mercado.

Mesmo alguns dos poucos estudos encontrados sobre marcas e desempenho não fazem menção ao desempenho de marca. Sugere-se para pesquisas futuras, investigar o desempenho de marca no mercado de alta tecnologia, bem como a construção de marcas fortes e inovação da marca neste mercado, uma vez que estudos relataram que este mercado ainda apresenta um maior foco no produto. Outro gap refere-se à identificação dos melhores e mais eficazes indicadores para se mensurar o desempenho mercadológico das empresas de alta tecnologia, pelo que é sugerido que novos estudos sejam realizados nesse sentido, ou seja, quais indicadores foram balizadores para as empresas de alta tecnologia de maior sucesso? Sugere-se também para pesquisas futuras, ampliar o quantitativo de bases de buscas (ex. bases latino-americanas), utilizar o método de efeito bola de neve para tentar identificar uma maior gama de estudos sobre a temática em próximas revisões.

Ademais, apesar das limitações do presente estudo, os resultados inferidos ratificam o potencial para novos estudos sobre desempenho mercadológico, especificamente no contexto do mercado de alta tecnologia, no sentido de contribuírem com a construção de um arcabouço 
teórico mais robusto para promover a ascensão do conhecimento teórico-empírico abordado, bem

\section{Referências}

Aaker, D. A. (1996), Measuring brand equity across products and markets. California Management Review, 38(3), 102-120

Aaker, D. A., \& Jacobson, R. (2001). The value relevance of brand attitude in high-technology markets. Journal of Marketing Research, 38(4), 485-493.

Ambler, T., \& Kokkinaki, F. (1997). Measures of marketing success. Journal of Marketing Management, 13(7), https://doi.org/10.1080/0267257X.1997.9964503

Ambler, T., Kokkinaki, F., \& Puntoni, S. (2004). Assessing marketing performance: reasons for metrics selection. Journal of Marketing Management, 20, 475-498. https://doi.org/10.1362/026725704323080506

Araújo, R. F., \& Alvarenga, L. (2011). A bibliometria na pesquisa científica da pós-graduação brasileira de 1987 a 2007. Revista Eletrônica de Biblioteconomia, 16(31), 51-70. https://doi.org/10.5007/1518-2924.2011v16n31p51

Atuahene-Gima, K., \& Li, H-Y. (2002). When does trust matter? Antecedents and contingent effects of supervisee trust on performance in selling new products in China and the United States. Journal of Marketing, 66(3), 61-81. https://doi.org/10.1509/jmkg.66.3.61.18501

Bayraktar, C. A., Hancerliogullari, G., Cetinguca, B., \& Calisir, F. (2016). Competitive strategies, innovation, and firm performance: an empirical study in a developing economy environment. Technology Analysis \& Strategic Management.

https://doi.org/10.1080/09537325.2016.1194973

Bruton, G. D., \& Rubanik, Y. (2002). Resources of the firm, Russian high-technology startups, and firm growth. Journal of Business Venturing 17, 553-576. https://doi.org/10.1016/S0883-9026(01)00079-9

Camargo, B. V., \& Justo, A. M. (2013). IRAMUTEQ: um software gratuito para análise de dados textuais. Temas Psicol., 21(2), 513-518. http://dx.doi.org/10.9788/TP2013.2-16

Camargo, B. V. (2005). ALCESTE: um programa informático de análise quantitativa de dados textuais. Perspectivas teórico-metodológicas em representações sociais, 1, 511-539.

Chang, S-C, Lin, N-P, \& Sheu, C. (2002). Aligning manufacturing flexibility with environmental uncertainty: evidence from high-technology component manufacturers in Taiwan. International Journal of como ampliar a possibilidade de futura generalização dos resultados.

Production Research, 40(18), 4765-4780.
https://doi.org/10.1080/00207540210157196

Chen, Y., Wang, Y., Nevo, S., Benitez, J., \& Kou, G. (2017). Improving strategic flexibility with information technologies: insights for firm performance in an emerging economy. Journal of Information Technology, 1-16. https://doi.org/10.1057/jit.2015.26

Chiesa, V., \& Frattini, F. (2011). Commercializing Technological Innovation: Learning from Failures in HighTech Markets. Journal of Product Innovation Management, 28, 437-454. https://doi.org/10.1111/j.15405885.2011.00818.x

Clark, B. (1999). Marketing performance measures: history and interrelationships. Journal of Marketing Management, 15(8), 711-32. https://doi.org/10.1362/026725799784772594

Clark, B. H., \& Ambler, T. (2001). Marketing performance measurement: evolution of research and practice. International Journal of Business Performance Management, 3, 231-244. https://doi.org/10.1504/IJBPM.2001.000101

Dawes, J. (1999). The Relationship between Subjective and Objective Company Performance Measures in Market Orientation Research: Further Empirical Evidence. Marketing Bulletin, 10, 65-75.

Dybå, T., \& Dings øyr, T. (2008). Strength of evidence in Systematic Reviews in software engineering. Proceedings of the Second ACM-IEEE international symposium on Empirical software engineering and measurement. Empirical Software Engineering and Measurement ESEM'08, 178-187. doi: 10.1145/1414004.1414034

Eusebio, R., Andreu, J. L., \& Belbeze, M. P. L. (2006). Measures of marketing performance: a comparative study from Spain. International Journal of Contemporary Hospitality Management, 18(2), 145-155. https://doi.org/10.1108/09596110610646691

Fuertes-Callén, Y., \& Cuéllar-Fernández, B. (2014). What is the role of commercialisation and reputation in product innovation success? Innovation: Management, Policy \& Practice, 16(1), 96-105. https://doi.org/10.5172/impp.2014.16.1.96

Gama, A. P. (2011). An expanded model of marketing performance. Marketing Intelligence \& Planning, 29(7), 643-661. https://doi.org/10.1108/02634501111178677

Gao, Y. (2010). Measuring marketing performance: a review and a framework. The Marketing Review, 10(1), 25-40. https://doi.org/10.1362/146934710X488924 
Greenhalgh, T. (1997). How to read a paper: Papers that summarise other papers (systematic reviews and metaanalyses). Bmj, 315, 672-675. https://doi.org/10.1136/bmj.315.7109.672

Guissoni, L. A., \& Neves, M. F. (2013). Ensaio sobre a análise de desempenho em marketing e aplicação de métricas. Revista Brasileira de Marketing, 12(4), 201229. doi: 10.5585/remark.v12i4.2535

Harris, L.C. (2001). Market orientation and performance: objective and subjective empirical evidence from uk companies. Journal of Management Studies 38(1), 18-43. https://doi.org/10.1111/1467$\underline{6486.00226}$

Kou, T-C, \& Lee, B. C. Y. (2015). The influence of supply chain architecture on new product launch and performance in the high-tech industry. Journal of Business \& Industrial Marketing, 30(5), 677-687. https://doi.org/10.1108/JBIM-08-2013-0176

Kou, T-C., Lee, B. C. Y., \& Wei, C-F. (2015). The role of product lean launch in customer relationships and performance in the high-tech manufacturing industry. Journal of Operations \& Production Management, 35(8), 1207-1223. https://doi.org/10.1108/IJOPM-08-2013-0397

Lau, C. M., \& Bruton, G. D. (2011). Strategic orientations and strategies of high technology ventures in two transition economies. Journal of World Business, 46, 371-380.

https://doi.org/10.1016/j.jwb.2010.07.011

Lew, Y. K., Sinkovics, R. R., \& Kuivalainen, O.(2013). Upstream internationalization process: Roles of social capital in creating exploratory capability and market performance. International Business Review, 22, 11011120. https://doi.org/10.1016/j.ibusrev.2013.03.001

Li, H., \& Atuahene-Gima, K. (1999). Marketing's Influence and New Product Performance in Chinese Firms. American Marketing Association, 7(1), 34-56.

Ma, C., Yang, Z., Yao, Z., Fisher, G., \& Fang, E. (2012). The effect of strategic alliance resource accumulation and process characteristics on new product success: Exploration of international high-tech strategic alliances in China. Industrial Marketing Management, 41, 469-480. https://doi.org/10.1016/j.indmarman.2011.04.001

McKee, D.O., Varadarajan, P.R., \& Pride, W.M. (1989). Strategic adaptability and firm performance. Journal of Marketing, 53(3), 21-35. doi: $10.2307 / 1251340$

Moghaddam, K., Bosse, D. A., \& Provance, M. (2016). Strategic Alliances of Entrepreneurial Firms: Value Enhancing Then Value Destroying. Strategic
Entrepreneurship Journal, 10, 153-168. https://doi.org/10.1002/sej.1221

Mohr, J., \& Shooshtari, N.H. (2003). Introduction to the special issue: marketing of high-technology products and innovations. Journal of Marketing Theory \& Practice, 11(3), 1-12. https://doi.org/10.1080/10696679.2003.11658497

Mohr, J. J., Sengupta, S. \& Slater, S. (2010). Marketing of high-technology products and innovations. 3 ed. Upper Saddle River, NJ: Prentice Hall.

Morgan, N. A., Vorhies, D. W., \& Mason, C. H. (2009). Market orientation, marketing capabilities, and firm performance. Strategic Management Journal, 30, 909920. https://doi.org/10.1002/smj.764

Mukarram, S. S., Saeed, A., Hammoudeh, S., \& Raziq, M. M. (2018). Women on Indian boards and market performance: a role-congruity theory perspective. Asian Business \& Management, 17(1), 4-36. https://doi.org/10.1057/s41291-018-0030-1

Nguyen, B., Yu, X., Melewar, T. C., \& Gupta, S. (2016). Critical brand innovation factors (CBIF): Understanding innovation and market performance in the Chinese high-tech service industry. Journal of Business Research, 69,

2471-2479.

https://doi.org/10.1016/j.jbusres.2016.02.016

Oh, C., Cho, Y., \& Kim, W. (2015). The effect of a firm's strategic innovation decisions on its market performance. Technology Analysis \& Strategic Management, 27(1), 39-53. https://doi.org/10.1080/09537325.2014.945413

O'Sullivan, D., \& Abela, A. V. (2007). Marketing Performance Measurement Ability and Firm Performance. Journal of Marketing, 71, 79-93. Retrieved from http://www.jstor.org/stable/30162185

O'Sullivan, D., Abela, A. V., \& Hutchinson, M. (2009). Marketing performance measurement and firm performance: Evidence from the European hightechnology sector. European Journal of Marketing, 43(5/6),

843-862. https://doi.org/10.1108/03090560910947070

Patel, C. (2014). Successful service retail channel expansions: The roles of technical and brand integration. Industrial Marketing Management, 43, 102-112.

https://doi.org/10.1016/j.indmarman.2013.07.020

Patterson, P. G., \& Dawes, P. L. (1999). The Determinants of Choice Set Structure in High-Technology. Industrial Marketing Management, 28, 395-411. https://doi.org/10.1016/S0019-8501(98)00030-3 
Pelham, A. M., \& Wilson, D. T. (1996). A longitudinal study of the impact of market structure, firm structure, strategy, and market orientation culture on dimensions of small-firm performance. Journal of the Academy of Marketing Science, 24(1), 27-43. https://doi.org/10.1007/BF02893935

Pereira, V. R., Carvalho, M. M. de, \& Rotondaro, R. G. (2013). Um estudo bibliométrico sobre a evolução da pesquisa da qualidade em serviço. Produção, 23(2), abr./jun., 312-328. http://dx.doi.org/10.1590/S0103$\underline{65132012005000053}$

Perin, M. G., \& Sampaio, C. H. (1999). Performance empresarial: uma comparação entre indicadores subjetivos e objetivos. Encontro Anual da Associação Nacional dos Programas de Pós-Graduação em Administração - EnAnpad, Foz do Iguaçu, PR, Brasil, 23.

Ratinaud, P. (2014). IRAMUTEQ: Interface de R pour les Analyses Multidimensionnelles de Textes et de Questionnaires - 0.7 alpha 2. Retrieved from: http://www.iramuteq.org.

Rauen, A. T., \& Furtado, A. T. (2014). Indústria de Alta Tecnologia: uma tipologia baseada na intensidade de P\&D e no desempenho comercial. Revista Brasileira de Inovação, 13(2), 405-432. https://doi.org/10.20396/rbi.v13i2.8649084

Reinert, M. (1990). Alceste une méthodologie d'analyse des données textuelles et une application: Aurelia De Gerard De Nerval. Bulletin of Sociological Methodology/Bulletin de Méthodologie Sociologique, 26(1), 24-54.

\section{https://doi.org/10.1177/075910639002600103}

Reinert, M. (1983). Une méthode de classification descendante hiérarchique : application à l'analyse lexicale par contexte. Cahiers de l'analyse des données, 8 (2), 187198. Retrieved from: http://www.numdam.org/item/CAD $1983 \quad 8 \quad 2 \quad 187$ o/

Rother, E. T. (2007). Revisão sistemática $X$ revisão narrativa. Acta Paulista de Enfermagem, 20(2), abr./jun., vvi. http://dx.doi.org/10.1590/S0103-21002007000200001

Santos, J. B., \& Brito, L. A. L. (2012). Toward a subjective measurement model for firm performance. BAR, Braz. Adm. Rev., 9, 95-117. http://dx.doi.org/10.1590/S1807-76922012000500007

Szymanski, D.M., Troy, L.C., \& Bharadwaj, S.G. (1995). Order of entry and business performance. An empirical synthesis and reexamination. Journal of Marketing, 59(4), 17-33. doi:10.2307/1252325

Troung, Y., Klink, R. R., Simmons, G., Grinstein, A, \& Palmer, M. (2017). Branding strategies for hightechnology products: The effects of consumer and product innovativeness. Journal of Business Research, 70,

85-91.

https://doi.org/10.1016/j.jbusres.2016.07.003

Vandenbroucke, E., Knockaert, M., \& Ucbasaran, D. (2016). Outside Board Human Capital and Early Stage High-Tech Firm Performance. Entrepreneurship Theory and Practice, 40(4), 759-779. https://doi.org/10.1111/etap.12141

Vanti, N. (2002). Da bibliometria à webometria: uma exploração conceitual dos mecanismos utilizados para medir o registro da informação e a difusão do conhecimento. Ciência da Informação, 31(2), 152-162. http://dx.doi.org/10.1590/S0100-19652002000200016

Varadarajan, P. R. (1986). Product diversity and firm performance: an empirical investigation. Journal of Marketing, 50(3), 43-57. doi:10.2307/1251584

Venkatraman, N., \& Ramanujan, V. (1986). Measurement of business performance in strategy research: a comparison of approaches. Academy of Management Review, 11(4), 801-814. Retrieved from http://www.jstor.org/stable/258398

Wall, T. D., et al. (2004). On the validity of subjective measures of company performance. Personnel Psychoology, 57, 95-118. https://doi.org/10.1111/j.1744-6570.2004.tb02485.x

Wang, D. H-M, Chen, P-H, Yu, T. H-K, \& Hsiao, C-Y. (2015). The effects of corporate social responsibility on brand equity and firm performance. Journal of Business Research, 68, 2232-2236. https://doi.org/10.1016/j.jbusres.2015.06.003

Wu, C-W. (2011). Global marketing strategy modeling of high tech products. Journal of Business Research, 64, 1229-1233.

https://doi.org/10.1016/j.jbusres.2011.06.028

Wu, P-H, \& Lin, C-P. (2016). Learning to foresee the effects of social identity complexity and need for social approval on technology brand loyalty. Technological Forecasting \& Social Change, 111, 188-197. https://doi.org/10.1016/j.techfore.2016.06.028 


\title{
Sobre os Autores:
}

Gislayne da Silva Goulart - Universidade Federal de Mato Grosso do Sul - UFMS, Mato Grosso do Sul, (Brasil). E-mail: gislayne.goulart@ufms.com Orcid id: https://orcid.org/0000-0002-0188-0965

Alessandra Ferrari Weber - Universidade de Brasília - UnB, Brasília, (Brasil). E-mail: aferrariweber@gmail.com Orcid id: https://orcid.org/0000-0002-1952-3958

Rafael Barreiros Porto - Universidade de Brasília - UnB, Brasília, (Brasil). E-mail: rbarreirosporto@gmail.com Orcid id: https://orcid.org/0000-0002-6699-2386

\section{MARKET PERFORMANCE IN THE HIGH-TECH MARKET: A SYSTEMATIC REVIEW}

\author{
Gislayne da Silva Goulart, Alessandra Ferrari Weber, Rafael Barreiros Porto
} Universidade Federal de Mato Grosso do Sul - UFMS Mato Grosso do Sul, (Brasil) Universidade de Brasília - UnB, Brasília, (Brasil)

\section{ARTICLE DETAILS}

\section{Article history:}

Received: 22 March 2019

Accepted: 25 September 2019

Available online January: 01 th 2020

\section{Double Blind Review System}

\section{Scientific Editor}

Ilan Avrichir

\section{Key words}

High-tech

Performance indicators

Dynamic markets

Iramuteq

\section{RESUMO}

Objective: To analyze the scientific production related to the market performance in the high-tech market from 1997 to 2019.

Method: Bibliometrics and systematic review methodologies were used. The search process was performed in the Web of Science and SPELL databases and resulted in the selection of 23 articles within the defined scope, among 82 studies. The selected studies were analyzed through descriptive statistics, Descending Hierarchical Classification (DHC) and Corresponding Factor Analysis (CFA).

Results: The results point to recent interest in measuring performance in the hightech market, especially in emerging markets such as Taiwan and China, with the use of multidimensional indicators of a financial and non-financial nature. Through the DHC and CFA analyses, we have been able to observe four Classes - 'Internal Abilities', 'Alliances', 'Downstream Focus' and 'Upstream Focus' - and these dominant areas indicate the interest associated with market performance in the high-tech market.

Originality/relevance: The study innovates by proposing a protocol to perform textual analysis of scientific articles with the aid of Iramuteq software, enabling the identification of different approaches of the studies and grouping by similarities. Contributions: We propose an agenda for future studies based on the results and gaps about performance and indicators used by companies in the high-tech market. From the managerial point of view, it is noteworthy that the most used indicators in this market are sales, market share and new product performance. 


\title{
RENDIMIENTO DE MARKETING EN EL MERCADO DE ALTA TECNOLOGÍA: UNA REVISIÓN SISTEMÁTICA
}

\author{
Gislayne da Silva Goulart, Alessandra Ferrari Weber, Rafael Barreiros Porto \\ Universidade Federal de Mato Grosso do Sul - UFMS, Mato Grosso do Sul, (Brasil) \\ Universidade de Brasília - UnB, Brasília, (Brasil)
}

\section{DETALLES DEL ARTÍCULO}

\section{Historia del Artículo:}

Recibido: 22 Marzo 2019

Aceptado: 25 Septiembre 2019

Disponible en línea: 01 de enero 2020

Double Blind Review System

Editor Científico

Ilan Avrichir

Palabras-clave:

Alta tecnología

Indicadores de desempeño

Mercados dinámicos

Iramuteq

\section{RESUMO}

Objetivo: Analizar la producción científica relacionada con el desempeño del mercado en el mercado de alta tecnología de 1997 a 2019.

Método: Las metodologías Bibliometría y revisión sistemática se utilizaron. El proceso de búsqueda se realizó en las bases de datos Web of Science y SPELL y resultó en la selección de 23 artículos dentro del alcance definido, entre 82 estudios. Los estudios seleccionados se analizaron mediante estadística descriptiva, clasificación jerárquica descendente (CHD) y análisis de factores de correspondencia (AFC).

Resultados principales: Los resultados apuntan al interés reciente en medir el desempeño en el mercado de alta tecnología, especialmente en mercados emergentes como Taiwán y China, utilizando indicadores multidimensionales, financieros y no financieros. A través de los análisis CHD y AFC, se formaron cuatro clases - 'Habilidades internas', 'Alianzas', 'Enfoque descendente' y 'Enfoque ascendente' - cuyos temas predominantes explican el foco de interés asociado con el desempeño del mercado en el mercado de alta tecnología.

Relevancia/Originalidad: El estudio innova al proponer un protocolo para realizar análisis textuales de artículos científicos con la ayuda del software Iramuteq, que permite la identificación de diferentes enfoques de los estudios y agrupación por similitudes.

Contribuciones: Se propone una agenda de estudios futuros basada en los resultados y las brechas en el desempeño y los indicadores utilizados por las empresas en el mercado de alta tecnología. Desde el punto de vista gerencial, es notable que los indicadores más utilizados en este mercado son las ventas, la participación en el mercado y el rendimiento de los nuevos productos.

\section{Para citar este artigo:}

Goulart, G., Weber, A., \& Porto, R. (2020). Desempenho Mercadológico no Mercado de Alta Tecnologia: Uma Revisão Sistemática. Internext, 15(1), 37-52. doi:http://dx.doi.org/10.18568/internext.v15i1.535 